\title{
Increased Alveolar and Plasma Gelatinases Activity during Postpump Syndrome: Inhibition by Inhaled Nitric Oxide
}

\author{
Bernard Hubert, MD, *† Eric Troncy, DV, PhD, DUn, $\neq$ Dominique Gauvin, BSc, * Rame Taha, PhD, * \\ Daniel Pang, DVM, MSc, $\neq$ Guy Beauchamp, PhD, $\neq$ Anna Radomski, MD, $\S \|$ \\ Marek W. Radomski, MD, $\$ \| \mathbb{I}$ and Gilbert A. Blaise, MD*
}

\begin{abstract}
Postpump syndrome is associated with systemic inflammation. Matrix metalloproteinases (MMP)-2 and -9 contribute to proinflammatory and platelet-activator reactions. Nitric oxide (NO) is involved in the regulation of MMPs. The objectives of our study were to investigate the intensity of inflammation induced by 3 different surgical procedures, the effects of inflammation on the activity of MMPs, and the regulation of inflammation by inhaled NO (20 ppm). Inhaled NO was initiated immediately after tracheal intubation and maintained for the total duration of the experiments. Thirty pigs were equally randomized into 6 groups [sham; sham + NO; cardiopulmonary bypass; bypass $+\mathrm{NO}$; bypass + lipopolysaccharide $(1 \mu \mathrm{g} / \mathrm{kg}$ for $50 \mathrm{~min})$; bypass + lipopolysaccharide $+\mathrm{NO}$ ] and animals were subjected to anesthesia and mechanical ventilation up to $24 \mathrm{~h}$. The levels of MMP-2 and MMP-9 in plasma and bronchoalveolar lavage were measured using zymography. Bypass resulted in a time-dependent rise in MMP activity, an effect potentiated by lipopolysaccharide. Inhaled NO attenuated the effects of bypass + lipopolysaccharide. These results confirm that MMP-2 and MMP-9 are associated with the inflammatory process causing the postpump syndrome. Preemptive and continuous administration of inhaled NO helps to prevent increased MMP-2 and MMP-9 activity.
\end{abstract}

Key Words: cardiopulmonary bypass, inflammation, lung, matrix metalloproteinases, NO gas

(J Cardiovasc Pharmacol ${ }^{\mathrm{TM}}$ 2006;48:71-78)

$\mathrm{M}$ atrix metalloproteinases (MMPs) are zinc-dependent endopeptidases, known for their ability to cleave 1 or several constituents of the extracellular matrix. Zymogen forms of the MMPs (pro-MMPs) are secreted in the matrix

Received and accepted July 5, 2006

From the *Department of Anesthesia, Centre Hospitalier de l'Université de Montréal, Canada; †Department of Anesthesia and Intensive Care Medicine, Centre Hospitalier Universitaire de Liege, Belgium; ‡Department of Veterinary Biomedicine, Université de Montréal, St-Hyacinthe, Canada; §Department of Pharmacology, University of Alberta, Edmonton, Canada; \|Department of Integrative Biology and Pharmacology, University of Texas-Houston; and 9 Institute of Molecular Medicine for the Prevention of Human Diseases, University of TexasHouston.

This work was supported by the Canadian Institutes of Health Research Reprints: Dr Gilbert A. Blaise, Laboratory of Anesthesia, Deschamps Pavilion, Room FS-1136, CHUM-Notre-Dame Hospital, 1560 Sherbrooke Street East, Montreal, H2L 4M1, QC Canada (e-mail: blaisegil@sympatico.ca). Copyright (C) 2006 by Lippincott Williams \& Wilkins from a large number of cells types such as epithelial, endothelial, or smooth muscle cells. ${ }^{1,2}$ Activation of the proMMPs in the local environment can result in discrete alterations in tissue architecture, and their main role is physiological tissue remodeling during wound repair and growth development. ${ }^{1}$ Several studies have shown that extracellular matrix degradation by MMPs, specifically MMP-9, is involved in the pathogenesis of a wide spectrum of cardiovascular disorders, including atherosclerosis, restenosis, cardiomyopathy, congestive heart failure, myocardial infarction, aortic aneurysm, and postpump syndrome. ${ }^{3-5}$ The major physiological inhibitors of the MMPs in vivo are $\alpha_{2}$ macroglobulin and the family of specific tissue inhibitors of MMPs (TIMPs), naturally occurring proteins specifically inhibiting these proteases and produced by many cell types. The TIMPs bind with high affinity in a 1:1 molar ratio to the catalytic site of active MMPs, resulting in loss of proteolytic activity, particularly TIMP-1 to 72-kd (MMP-2) and 92-kd (MMP-9) gelatinases. Moreover, TIMP-1 and TIMP-2 can form a specific complex with pro-MMP-9 and pro-MMP-2, respectively. This interaction has been suggested to provide an extra level of regulation by potentially preventing activation. The production of gelatinase is controlled by a variety of agents, including proinflammatory cytokines such as interleukin- $1 \beta$ and tumor necrosis factor- $\alpha{ }^{6}$

Since the early days of cardiac surgery, it has been recognized that cardiopulmonary bypass (CPB) is associated with systemic inflammation, occasionally leading to major organ dysfunction. When organ dysfunction cannot be directly attributed to a specific cause, such as infection or ischemia, the concept of the "postpump syndrome" or "systemic inflammatory response syndrome to CPB" is used as an alternative explanation. ${ }^{7}$ Reduced arterial oxygenation is a common complication of the postpump syndrome. ${ }^{8}$ In a study of 400 patients undergoing a variety of cardiac surgeries with $\mathrm{CPB}$, there was a $40 \%$ decrease in dynamic lung compliance within the first $4 \mathrm{~h}$, and in alveolar-arterial oxygen gradient (from 296 at intensive care unit arrival to 152 at $12 \mathrm{~h}$ and $181 \mathrm{mmHg}$ at $24 \mathrm{~h}$ ). ${ }^{9}$ Acute respiratory distress syndrome (ARDS), the most severe form of lung dysfunction, with $\mathrm{PaO}_{2} / \mathrm{FiO}_{2}$ ratio below $200 \mathrm{mmHg}$, has an incidence rate after $\mathrm{CPB}$ of $0.5 \%$ to $1.7 \%$, with mortality reported to be between $40 \%$ and $60 \%$ or higher., ${ }^{7,10}$

A number of studies have associated the release of gelatinases with $\mathrm{CPB}$ and pulmonary injury. ${ }^{4,11-14}$ During and after CPB in humans, these MMPs are synthesized and released in plasma ${ }^{11-13}$ and in heart tissue (right atrial biopsy 
specimens). ${ }^{13}$ In addition, MMP-2 and MMP-9 levels are increased in bronchoalveolar lavage (BAL) of ARDS patients. ${ }^{14}$ Under experimental conditions, isolated rat hearts submitted to ischemia reperfusion have highlighted the deleterious role of MMP-2 in the recovery of mechanical function during reperfusion. ${ }^{15}$ In an animal model of CPBinduced acute lung injury (ALI), the importance of MMPs (-2 and -9) was demonstrated when all pathological changes typical of ALI after CPB were prevented by using a chemically modified tetracycline, a potent MMP and elastase inhibitor. ${ }^{4}$

Usually, nitric oxide (NO) inhalation improves hypoxemia in ALI/ARDS patients. ${ }^{16}$ The mediator NO has been suggested to have anti-inflammatory properties. It inhibits the release of cytokines and prevents the expression of adhesion molecules in endothelial cells, smooth muscle cells, leukocytes, and platelets. ${ }^{17,18}$ The administration of a NO donor ( $S$-nitroso-glutathione) during $\mathrm{CPB}$ reduced the $\mathrm{Ca}^{2+}$-independent activity of NO synthase (NOS) ${ }^{19}$ Furthermore, NO donors and prostacyclin inhibited the secretion of gelatinase A (MMP-2) induced by collagen and thrombin in intact platelets, indicating that enzyme release is controlled by both compounds. $^{20}$ However, preemptive infusion with an NO scavenger decreased gelatinases activities in the lung, left ventricle, and atrium in a canine CPB model. ${ }^{21}$ With the use of nonselective NOS and soluble guanylate cyclase inhibitors, it was suggested that NO and cyclic guanosine monophosphate are necessary to upregulate the expression of MMP-9. ${ }^{22}$

Our objectives in this study were first to measure the activity of MMP-2 and MMP-9 in sham-operated pigs and in animals subjected to $\mathrm{CPB}$ in the presence or absence of lipopolysaccharide (LPS). ALI/ARDS after CPB can develop after consecutive minor insults, with $\mathrm{CPB}$ acting as the initial inflammatory event. ${ }^{23} \mathrm{~A}$ short period of $\mathrm{CPB}$ alone is rather innocuous, but when combined with a subsequent, seemingly benign insult (ie, transient hypoxia, ischemia, endotoxemia with a low dose of LPS), the result is an overwhelming inflammatory response leading to endothelial injury, pulmonary edema, and ALI/ARDS. ${ }^{24}$ The second objective was to examine whether preemptive administration of inhaled NO exerts significant effects on the activity of MMPs in our anesthetized and mechanically ventilated porcine model of extracorporeal circulation. $^{25}$

\section{METHODS}

\section{Animals and Experimental Protocol}

This study was performed with the approval of the institutional animal care committee in compliance with Canadian Council on Animal Care guidelines. Thirty acclimated, 4month-old hybrid (Pietrain $\times$ Landrace) castrated male pigs $(37.2 \pm 2.5 \mathrm{~kg})$ free from clinically evident pulmonary disease were randomized into 6 groups of 5 animals. The first (sham) group was submitted to sternotomy with a 90-min pericardial opening, followed by closure. The second (CPB) group was subjected to 90-min CPB with 75-min aortic clamping. The third (CPB + LPS) group was subjected to the CPB procedure as above, plus receiving an infusion of $1 \mu \mathrm{g} / \mathrm{kg}$ of Escherichia coli LPS O111:B4 (Sigma-Aldrich, Stockholm, Sweden) mixed in $100 \mathrm{~mL}$ of isotonic saline and delivered at a flow rate of $120 \mathrm{~mL} / \mathrm{h}$ for $50 \mathrm{~min}$ commencing $1 \mathrm{~h}$ postoperatively. ${ }^{23}$ Fourth (sham + inhaled NO), fifth (CPB + inhaled NO), and sixth (CPB + LPS + inhaled NO) groups were submitted to the same procedure as their respective controls plus the administration of $20 \mathrm{ppm}$ inhaled NO, initiated immediately after induction of anesthesia and maintained for the surgery and the whole 24-h follow-up period.

Experimental setup, ventilation strategy, CPB, and inhaled NO administration (20 ppm) were performed as previously described by our group. ${ }^{25}$ We have selected a dose of inhaled NO (20 ppm) because this dose causes significant pulmonary vasodilatation ${ }^{26}$ and ensures an inspired fraction of $\mathrm{NO}_{2}$ below $1 \mathrm{ppm}$ with our synchronized intermittent mandatory ventilation system of NO administration. ${ }^{27}$

Pigs were premedicated intramuscularly with atropine $(0.04 \mathrm{mg} / \mathrm{kg})$, azaperone $(4 \mathrm{mg} / \mathrm{kg})$ and ketamine $(25 \mathrm{mg} / \mathrm{kg})$, and anesthesia induced with intravenous (auricular vein), fentanyl $(5 \mu \mathrm{g} / \mathrm{kg})$, and thiopental $(5 \mathrm{mg} / \mathrm{kg})$. After intubation with an 8-mm ID endotracheal tube (Mallinckrodt, Mexico City), the pigs were placed in a supine position. Anesthesia was maintained by continuous infusion of thiopental $(5 \mathrm{mg} / \mathrm{kg} / \mathrm{h})$ and fentanyl $(20 \mu \mathrm{g} / \mathrm{kg} / \mathrm{h})$. Muscle relaxation was induced with $0.2 \mathrm{mg} / \mathrm{kg}$ pancuronium with intermittent reinjection $(0.1$ $\mathrm{mg} / \mathrm{kg}$ ) to achieve optimal surgical and ventilatory conditions. After endotracheal intubation, 20 ppm of NO gas was injected cyclically into the inspiratory line during the inspiratory phase by a NO injector for $24 \mathrm{~h}$. A 1000-ppm NO balanced $\mathrm{N}_{2}$ cylinder was obtained from VitalAire Santé Ltd. (Montreal). The $\mathrm{NO}$ and $\mathrm{NO}_{2}$ concentrations delivered to the animals were monitored with an electrochemical device (Polytron $\mathrm{NO} / \mathrm{NO}_{2}$, Drager A.G., Lubeck, Germany). During CPB, NO was added directly to the gas mixture delivered to the oxygenator. Hemodynamic and respiratory monitoring was constant and for the whole $(24 \mathrm{~h})$ duration of the experiment. ${ }^{28}$

\section{CPB Materials and Procedure}

A median sternotomy was performed, and at the time of pericardial opening an intravenous injection of heparin (4 $\mathrm{mg} / \mathrm{kg}$ ) was given to achieve an activated clotting time $>400 \mathrm{~s}$. An aortic cannula (20 F, Chase Medical, Richardson, TX) was placed in the aortic root followed by placement of a multiple hole venous drainage cannula in the caudal vena cava via the right auricular appendage. A cardioplegia cannula (9 F, Medtronic, Grand Rapids, MI) was then placed in the aortic root proximal to the aortic valve, enabling cold blood cardioplegia. The CPB circuit consisted of a membrane oxygenator (Trillium Affinity NT oxygenator, Medtronic), a cardiotomy reservoir (Affinity NT541, Medtronic), a filter (Affinity 351, Medtronic), tubing, and a Sarns roller pump (Sarns, Ann Arbor, MI). The circuit was primed with $1500 \mathrm{~mL}$ lactated Ringer's solution (Baxter, Toronto), $500 \mathrm{~mL}$ Pentaspan colloidal fluid (DuPont Pharma, Mississauga, Canada), $1 \mathrm{mEq} / \mathrm{kg}$ sodium bicarbonate, $5000 \mathrm{IU}$ heparin, and $200 \mathrm{~mL}$ mannitol. Cardiopulmonary bypass was initiated at a flow rate of $3.0 \mathrm{~L} / \mathrm{min} / \mathrm{m}^{2}$ and blood temperature was decreased to $32^{\circ} \mathrm{C}$. Following aortic clamping, cardiac arrest was induced by continuous hyperkaliemic cold blood cardioplegia $\left(8^{\circ} \mathrm{C}-12^{\circ} \mathrm{C}\right)$ through the aortic cardioplegia cannula at a flow rate of $500 \mathrm{~mL} / \mathrm{min}$, not exceeding a perfusion pressure of 
$100 \mathrm{mmHg}$. Heart temperature measured by a temperature probe placed in the left ventricular myocardium was maintained below $15^{\circ} \mathrm{C}$ throughout the procedure. Pulmonary capillary wedge pressure was kept $<15 \mathrm{mmHg}$ by intermittent interruption of cardioplegia and cardiac decompression through the cardioplegia aortic cannula. Mean systemic arterial blood pressure was maintained between 50 and $65 \mathrm{mmHg}$ by adjustment to the flow rate, plus a bolus of phenylephrine [0.5-1 $\mathrm{mL}(0.1 \mathrm{mg} / \mathrm{mL})]$, if required. Arterial partial pressure of carbon dioxide $\left(\mathrm{PaCO}_{2}\right)$ was maintained between 40 and $50 \mathrm{mmHg}$ by adjusting fresh gas flow. The aorta was clamped for $75 \mathrm{~min}$. Blood rewarming commenced 10 min before aortic declamping using water at $38^{\circ} \mathrm{C}$. Following aortic clamp removal, the heart was electrically defibrillated $(20 \mathrm{~J})$ as ventricular fibrillation is observed in most pigs after reperfusion. ${ }^{25}$ The cardioplegia was stopped and the animals were subsequently weaned from $\mathrm{CPB}$ once rectal temperature was $>35^{\circ} \mathrm{C}$. The total length of CPB was at least 90 min. Intravenous protamine (1 mg/100 IU heparin) normalized activated clotting time. Hemostasis was performed after removal of the CPB cannulae, thoracic drainage placed under negative pressure, and the chest was closed. Blood from the CPB circuit was subsequently transfused.

At T0 (just before chest opening), T4 ( $2 \mathrm{~h}$ post-CPB), and T24 (22 h post-CPB), blood and BAL samples were collected for the measurement of MMPs. Fiberoscopy was undertaken for BAL with isotonic sterile saline solution injected as 3 aliquots of $25 \mathrm{~mL}$. Lavage was performed at T0 in the right accessory lobe, T4 in the lower left lobe, and T24 in the lower right lobe. The total recovered BAL samples (kept at $4^{\circ} \mathrm{C}$ throughout the procedure) were filtered and centrifuged for $8 \mathrm{~min}$ at $150 \mathrm{~g}$ to separate the surfactant (supernatant) from cells and cellular debris. The supernatant was frozen at $-80^{\circ} \mathrm{C}$ for further analysis of MMPs. Total cell count in BAL was achieved by the hemocytometer method.

\section{MMP-2 and MMP-9 Analysis}

The release of MMP-2 and MMP-9 during CPB was measured by zymography as described previously. ${ }^{20,31}$ This method has been validated for the MMP measurement in humans and in a canine model of CPB. ${ }^{13,21}$ Briefly, zymography is performed by subjecting samples (20 $\mu \mathrm{g}$ protein each) to $8 \%$ sodium dodecyl sulfate-polyacrylamide gel electrophoresis with copolymerized gelatin $(2 \mathrm{mg} / \mathrm{mL}$, Sigma, St Louis, MO) as a substrate. After electrophoresis, the gels are washed with $2 \%$ Triton X-100 and incubated in buffer $(50 \mathrm{mmol} / \mathrm{L}$ Tris- $\mathrm{HCl}$ buffer with $0.15 \mathrm{~mol} / \mathrm{L} \mathrm{NaCl}, 5 \mathrm{mmol} / \mathrm{L} \mathrm{CaCl}{ }_{2}$, and $0.05 \%$ $\mathrm{NaN}_{3}, \mathrm{pH} 7.5$ ) at $37^{\circ} \mathrm{C}$ until the activities of the enzymes can be determined. After incubation, the gels are stained with $0.05 \%$ Coomassie brilliant blue G-250 (Sigma) in a mixture of methanol:acetic acid:water (2.5:1:6.5) and destained in $4 \%$ methanol with $8 \%$ acetic acid. The gelatinolytic activities are detected as transparent bands against the background of Coomassie brilliant blue-stained gelatin. Enzyme activities are quantified using a gel documentation system (Bio-Rad) and expressed as specific activity per milligram of proteins. ${ }^{20,31}$

\section{Statistical Analysis}

For the estimation of necessary sample size, we used published results from human ${ }^{4,11-14}$ and canine models. ${ }^{19,21}$
These data suggested that a minimum population size of 5 per group would detect a significant difference $80 \%$ of the time with an $\alpha$ level of 5\% for the related level of MMPs released during postpump syndromes and its possible inhibition by inhaled NO. The comparison of MMP-2 and -9 activities between experimental groups was done using Kruskal-Wallis and Mann-Whitney $U$ tests with a correction for post hoc pairwise analyses. Time courses of this release were compared using the Friedman test. Where significance was found in this intragroup comparison, a Wilcoxon test was used for post hoc analysis, followed by Dunn's correction. The level of statistical significance was set at 0.05 before correction and data are presented as mean \pm standard deviation.

\section{RESULTS}

\section{Intergroup Comparison}

In plasma, the rise of MMP-2 activity occurred at time T24 in pigs submitted to CPB vs sham $(P<0.05$; Fig. 1A, B $)$ and to CPB + LPS vs sham at time T4 $(P<0.01$; Fig. $1 \mathrm{~A})$ and T24 $(P<0.05$; Fig. 1B). The rise of MMP-9 activity was significantly higher after CPB + LPS vs sham and CPB at time T4 $(P<0.01$; Fig. 2A). The difference was no longer present at time $\mathrm{T} 24$.

In BAL, MMP-2 activity increased significantly in pigs submitted only to CPB + LPS vs sham at time T4 $(P<0.01$; Fig. 1C) and at time T24 $(P<0.01$; Fig. 1D). The rise of MMP-9 activity was significantly higher in pigs submitted to CPB + LPS vs sham $(P<0.01)$ at time T24 (Fig. $2 D)$.

\section{Time-dependent Effect of the Insult on MMPs for Each Group}

In BAL and plasma, there was no difference in time for MMP-2 and MMP-9 in the sham group without or with inhaled NO. The MMP-2 activity increased in BAL over time in the CPB group at time T4 compared with time T0 (not significant) and at time T24 vs time T0 $(P<0.01$; Fig. 3$)$; this effect was also present in the CPB + LPS group $(P<0.05$ at time T24 vs time T0; Fig. 4). Activity of MMP-9 increased significantly over time in BAL in the CPB group at time T24 vs time T0 $(P<0.05)$ and in CPB + LPS group at time T24 vs time T4 $(P<0.05)$.

In plasma, MMP-2 also demonstrated an increased activity over time in both CPB $(P<0.01$ at time T24 vs time T0; Fig. 3$)$ and CPB + LPS groups $(P<0.01)$ at time T24 vs time T0; Fig. 4). In plasma, the rise of MMP-9 activity occurred in the CPB + LPS group at time T4 vs T0 $(P<0.05)$; MMP-9 activity then returned to the initial value at time T24.

\section{Effect of the Procedure}

The sham procedure did not induce any change in MMPs. The CPB alone induced an increase of MMP-2 activity in plasma and BAL at T24 and of MMP-9 only in BAL at T24. This was corroborated by the statistically significant comparison between sham and CPB groups at T24 for MMP-2 in plasma. The CPB + LPS procedure similarly induced an increase of MMP-2 and MMP-9 activity in BAL at T24 and of MMP-2 in plasma at the same time. Moreover, an increase in MMP-9 was observed in plasma for this group at T4, but was 
Effects of Inhaled NO on MMP-2 activity in plasma at time T4

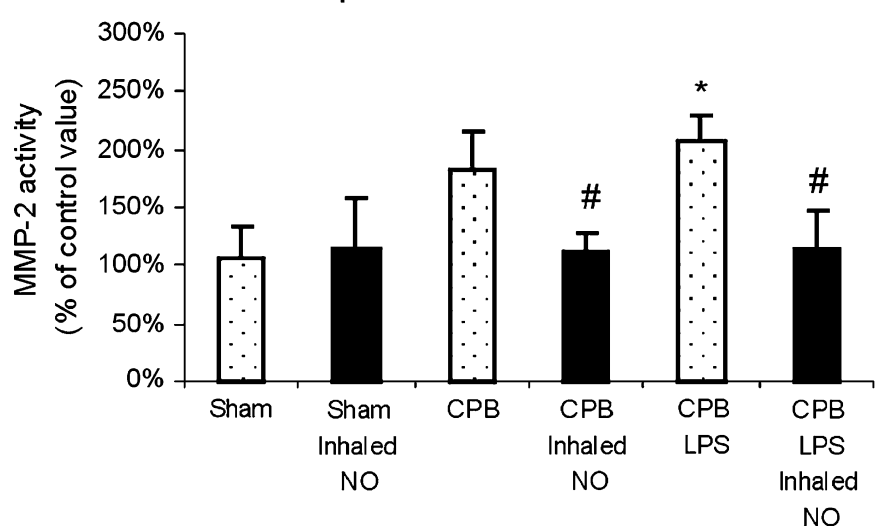

Effects of Inhaled NO on MMP-2 activity in BAL at time T4

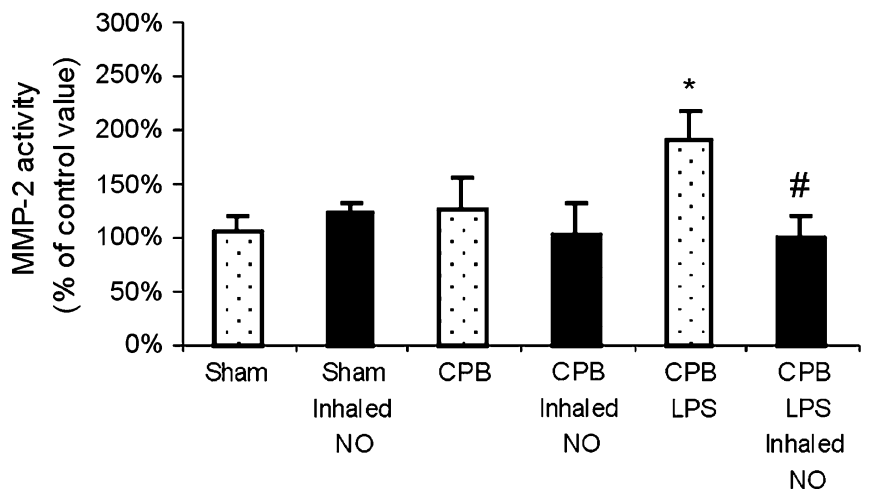

Effects of Inhaled NO on MMP-2 activity in plasma at time T24

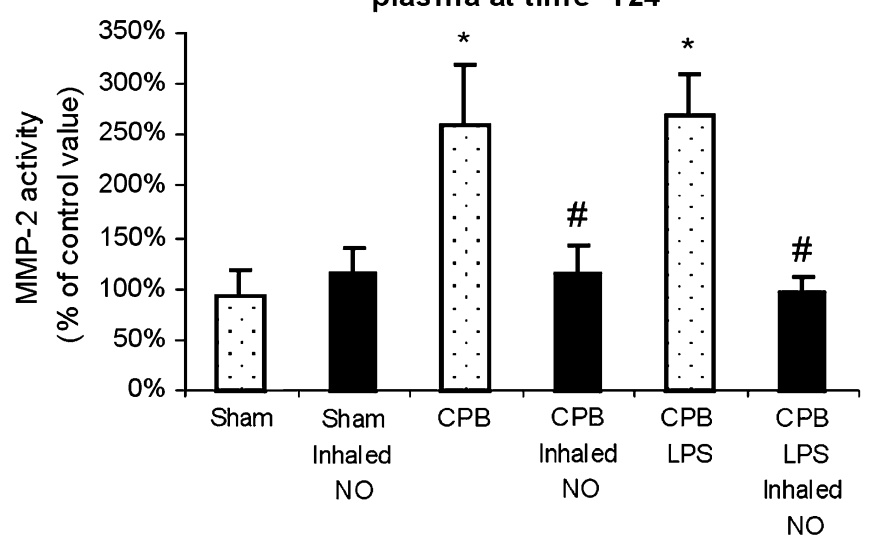

Effects of Inhaled NO on MMP-2 activity in BAL at time T24

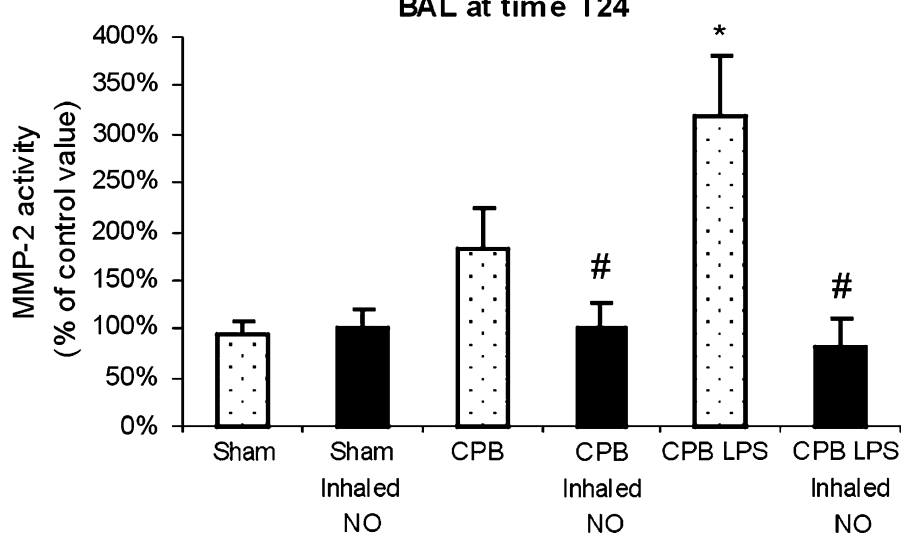

FIGURE 1. Variation of MMP-2 activity in BAL and plasma at time T4 and T24 vs T0 (\%). Intergroup comparison * $P<0.05$ vs sham, $\# P<0.05$ vs group without inhaled NO.

no longer present at T24. This was corroborated by the statistically significant comparison between sham and CPB + LPS groups at T4 and T24 for MMP-2 in plasma and BAL, and for MMP-9 in plasma at T4 and in BAL at T24.

\section{Effect of Inhaled NO}

In the sham group, MMP-2 and MMP-9 activities were not changed with inhaled NO both in BAL and in plasma at time T4 and time T24. In the CPB group, the rise of MMP-2 activity in BAL was significantly higher compared to $\mathrm{CPB}+$ inhaled NO group at time T24 $(P<0.05$; Fig. 1D). In plasma, the rise of MMP-2 activity was also significantly higher in the CPB group compared to CPB + inhaled NO at time T4 $(P<$ 0.05 ; Fig. 1A) and time T24 $(P<0.05$; Fig. 1B). These differences were not found for MMP-9 activities in both BAL and plasma.

In the CPB + LPS group, the rise of MMP-2 activity in BAL was significantly higher in the CPB + LPS group compared to CPB + LPS + inhaled NO at time T4 $(P<0.05$; Fig. 1C) and time T24 $(P<0.05$; Fig. 1D). This effect was significant for MMP-9 activity in BAL only at time T24 $(P<$ 0.05 ; Fig. 2D). In plasma, the rise of MMP-2 activity was significantly higher in the CPB + LPS group compared to $\mathrm{CPB}+\mathrm{LPS}+$ inhaled NO group at time T4 $(P<0.05$; Fig. $1 \mathrm{~A})$ and T24 $(P<0.05$; Fig. 1B). This effect was not found for MMP-9.

\section{Cell Count in BAL and Leukocyte Count in Blood}

There was no change in the BAL cell count over time either in the 3 inhaled NO-treated groups or in the sham group. However, at T24, the BAL cell count was statistically higher compared to T0 in both CPB $(P=0.007)$ and $\mathrm{CPB}+$ LPS $(P=0.02)$ groups. The increased value at T24 in CPB and $\mathrm{CPB}+$ LPS groups was higher than the value in the sham group $(P<0.03)$. The difference between control groups and inhaled NO-treated groups did not reach statistical significance $(P=0.09)$ at T24.

Moreover, the neutrophil differentials in BAL were also greatly augmented $(P<0.05)$ at T24 in the CPB $(206 \%)$ and CPB + LPS (244\%) groups, but were lower in the inhaled NOtreated animals without reaching statistical significance. No similar difference could be observed for monocytes or lymphocytes in BAL. The CPB + LPS procedure had a significant 
Effects of Inhaled NO on MMP-9 activity in plasma at time T4.

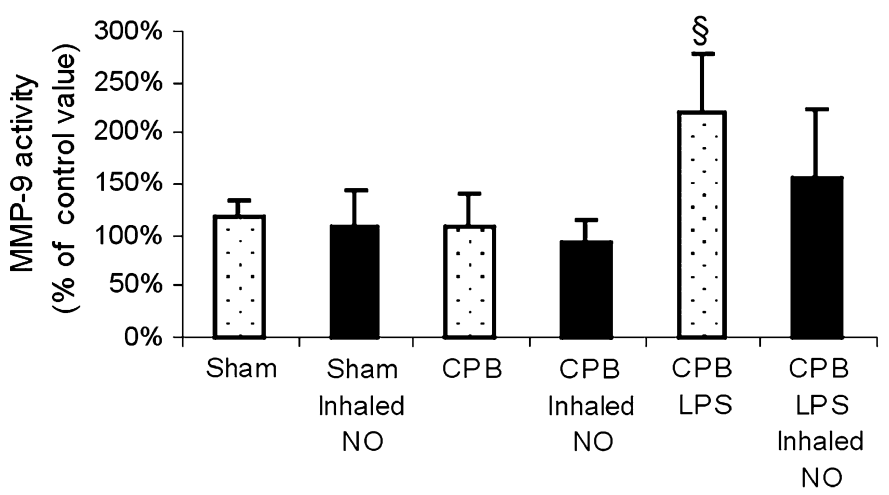

Effects of Inhaled NO on MMP-9 activity in BAL at time T4

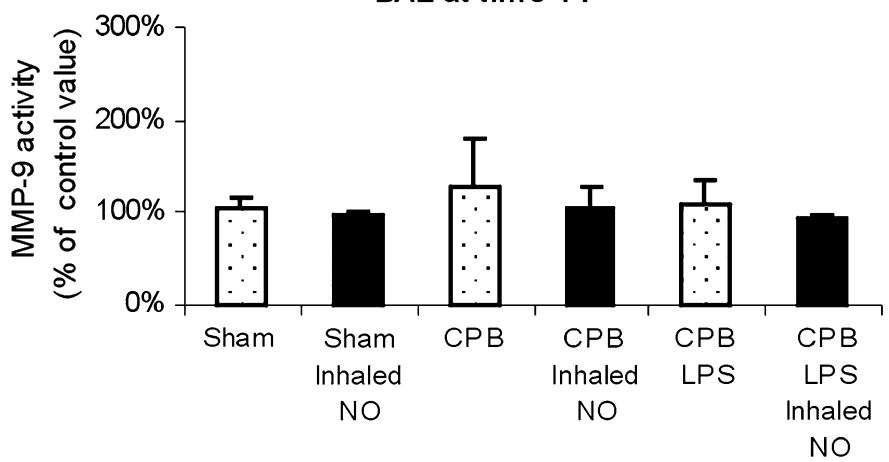

Effects of Inhaled NO on MMP-9 activity in plasma at time T24

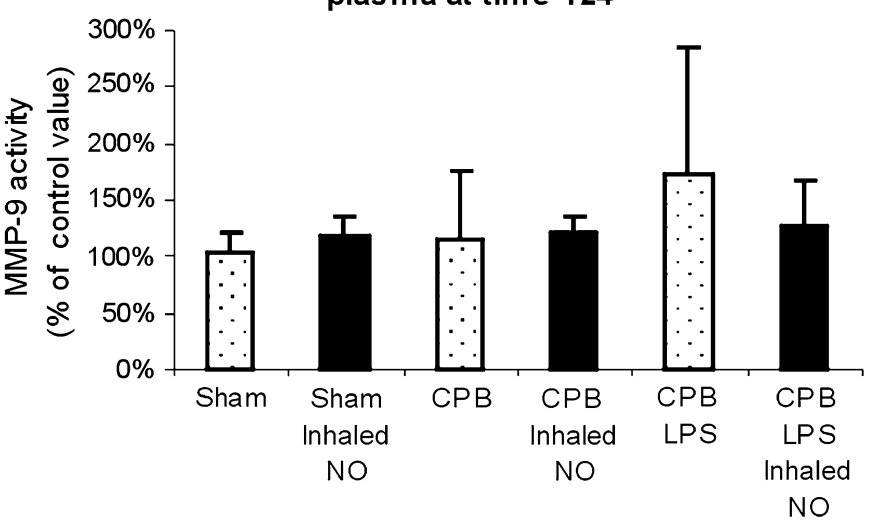

Effects of Inhaled NO on MMP-9 activity in BAL at time T24

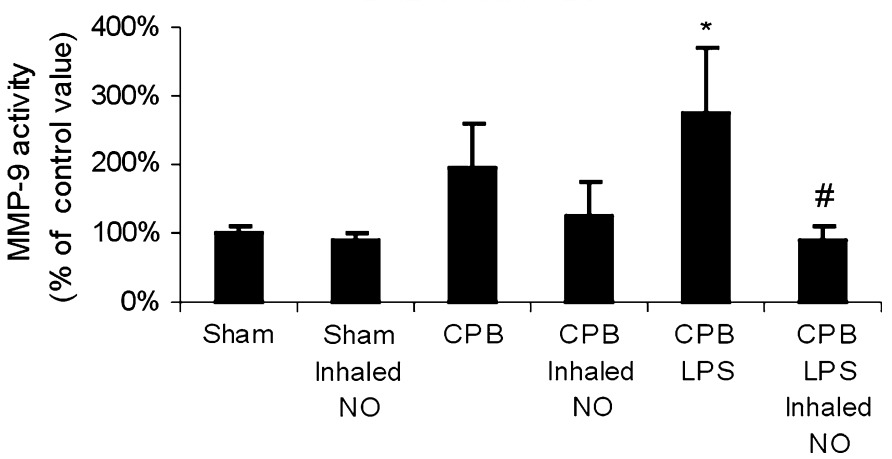

FIGURE 2. Variation of MMP-9 activity in BAL and plasma at time T4 and T24 vs T0 (\%). Intergroup comparison * $P<0.05$ vs sham, $\S P<0.05$ vs $C P B, \# P<0.05$ vs group without inhaled NO.

effect on the blood leukocyte count over time; whereas it decreased with time in the sham and CPB $(P=0.004)$ groups, it increased in the CPB + LPS group. In the sham and CPB groups, leukocyte count decreased over time, respectively, from $18.9 \pm 5.1 \times 10^{3} / \mathrm{mm}^{3}$ at time T0 to $13.7 \pm 1.6 \times$ $10^{3} / \mathrm{mm}^{3}$ at time T24 and $22.4 \pm 5.5 \times 10^{3} / \mathrm{mm}^{3}$ at time T0 to $13 \pm 1.6 \times 10^{3} / \mathrm{mm}^{3}$ at time T24. In the CPB + LPS group, the leukocyte count increased from $16.7 \pm 3.5 \times 10^{3} / \mathrm{mm}^{3}$ at time T0 to $18.9 \pm 7.3 \times 10^{3} / \mathrm{mm}^{3}$ at time T24. Moreover, inhaled NO had no effect on blood leukocyte count over time.

\section{DISCUSSION}

The postpump syndrome constitutes a model of systemic and local inflammatory reactions with cellular (leukocytes, platelets, endothelial cells) activation and the release of proinflammatory cytokines and enzymes mediating acute inflammation and organ injury. ${ }^{7,24}$ We have developed a porcine model to study the gelatinase activity after CPB and its potential implication in the associated inflammatory response.

Because CPB constitutes only an initial stimulus not systematically associated with consequences, inflammatory reaction was potentiated with subsequent LPS infusion. At $1 \mu \mathrm{g} / \mathrm{kg}$ for $50 \mathrm{~min}$, LPS in association with CPB increases the inflammatory reaction to induce a real postpump syndrome, and at this dose it does not induce ALI alone. ${ }^{4,23,24}$
Inhaled NO decreases neutrophil and platelet activation and inhibits neutrophil function under ischemia/reperfusion ${ }^{32}$ and $\mathrm{ARDS}^{33}$ conditions. Moreover, combined therapy with NO gas (20 ppm) and iloprost (a stable analog of prostacyclin, $2 \mathrm{ng} / \mathrm{kg} / \mathrm{min}$ ) reduced the deleterious effects of CPB in human patients, such as thrombocytopenia, platelet activation, plateletleukocyte aggregate formation, and suppression of platelet aggregative responses. ${ }^{34}$ This result was in agreement with previous demonstration of a possible beneficial effect of NO donors to inhibit the $\mathrm{Ca}^{2+}$-independent activity of iNOS increased after $\mathrm{CPB}^{19}$ or the secretion of MMP-2. ${ }^{20}$ At the same time, inhibition of the NO pathway (with an NO scavenger and a NOS inhibitor, respectively) decreased the MMP-2 and -9 activities after $\mathrm{CPB}^{21}$ and prevented the upregulation of MMP-9, ${ }^{22}$ possibly by altering the induction of NOS activity. NO appears to exert dual effects in CPB. When generated by iNOS, NO, probably through increased generation of peroxynitrite, contributes to the development of CPB-induced inflammation. In contrast, pharmacologically provided $\mathrm{NO}$, in the form of inhaled $\mathrm{NO}^{34}$ or NO donor, ${ }^{19,20}$ has been shown to attenuate the inflammatory lesions induced by extracorporeal circulation.

Because neutrophils and platelets are implicated in the physiopathology of postpump lung syndrome and release of gelatinases, we have studied the effect of inhaled NO on MMP-2 and -9 activity in plasma and BAL, on neutrophil numbers and their major chemoattractant (interleukin-8), and 


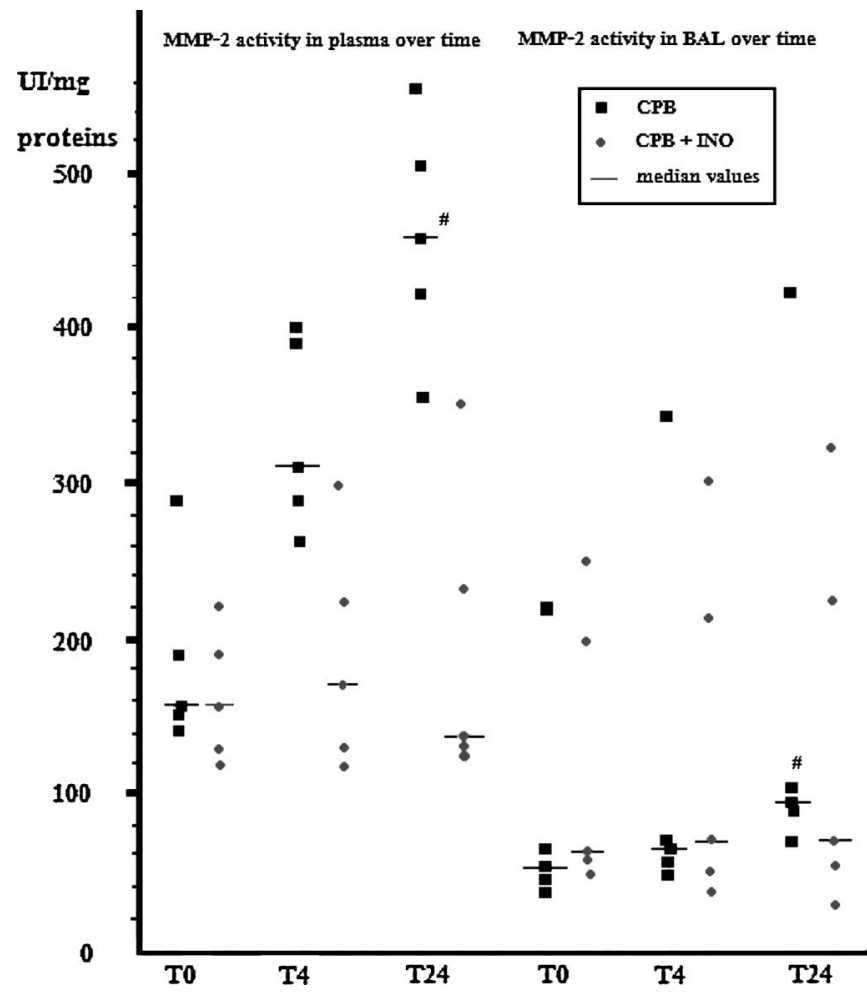

FIGURE 3. MMP-2 activity (individual data) in plasma and BAL in CPB groups (with and without inhaled NO treatment) over time (UI/mg proteins). \#P $<0.05$ vs T0.

in pig submitted to sham operation, CPB, and CPB + LPS. We hypothesized that the postpump syndrome could be induced by an initial deficiency in endogenous NO in relationship to the nonpulsatile blood flow generated by the CPB machine, decreasing the shear stress source of endothelial NOS stimulation. Deficient NO production affects the inflammatory cascade, allowing the vascular adhesion of inflammatory cells primed by contact with the extracorporeal circuit. Indeed, we have observed with the same model decreased levels of nitrite, nitrate (NOx) stable plasma metabolites of the endogenous NO production in all 3 groups treated without inhaled NO (sham, CPB, CPB + LPS). ${ }^{29,30}$ This was not the case in comparative groups treated with preemptive 20 -ppm inhaled NO in swine ${ }^{29,30}$ and in human patients. ${ }^{35}$ This initial deficit in endothelial NO synthesis is particularly marked in older patients and could be judiciously replaced by a preemptive administration of exogenous NO donor ${ }^{19}$ or inhaled $\mathrm{NO}^{29,30,35}$ at a low dose. The preemptive use of a NO donor suppressed the increased $\mathrm{Ca}^{2+}$ independent activity of iNOS observed after $\mathrm{CPB},{ }^{19}$ and the inhibition of the NO pathway decreased gelatinases activity after CPB. ${ }^{21}$ Therefore, we speculated that NO replacement therapy could blunt the consequences of induction of NOS activity and systemic inflammation, ${ }^{35}$ leading to the decreased activity of gelatinases.

The release of MMPs during CPB appears to be dependent on the intensity (as reflected by the initial inflammatory insult induced by CPB and amplified by repeated subsequent stimuli such as mechanical ventilation or LPS perfusion) of the inflammatory stimuli. Indeed, in BAL and plasma, the levels

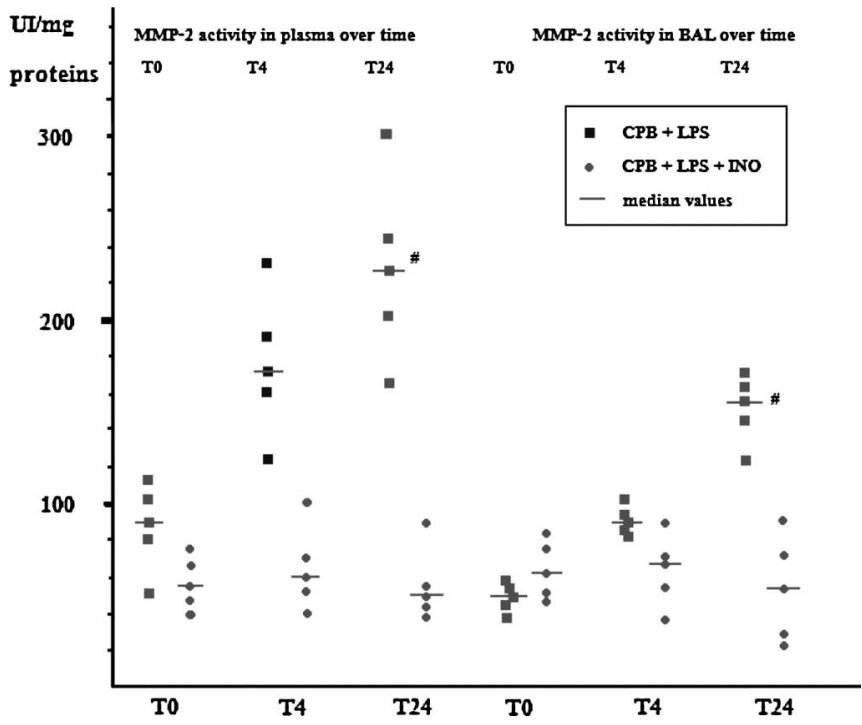

FIGURE 4. MMP-2 activity (individual data) in plasma and BAL in CPB + LPS groups (with and without inhaled NO treatment) over time (UI/mg proteins). \#P<0.05 vs T0.

of MMP-2 and -9 remained stable in the sham group during the 24-h period. In pigs submitted to CPB, MMP-2 activity increased only in plasma after $24 \mathrm{~h}$, compared to the sham group. This could reflect the summation of inflammatory stimuli induced initially by CPB and repeated by the ventilator-induced lung injury under general anesthesia. In pigs submitted to $\mathrm{CPB}+$ LPS, the increase in MMP-2 activity occurred both in plasma and BAL immediately after the end of LPS administration (at T4) and for a period of $22 \mathrm{~h}$, and was significantly higher than in the sham group. The rise of MMP-9 activity was significantly higher in plasma after LPS infusion (at T4) compared to the CPB group, but only occurred in BAL after $24 \mathrm{~h}$ compared to the sham group. We can conclude that the LPS administration after CPB constitutes a potent stimulus for the rise of the MMP activities. Our data confirm the results described by Carney et $\mathrm{al}^{4}$ and Picone et al. ${ }^{23}$ Interestingly the CPB + LPS group was the only group that developed severe physiological lung injury typical of ARDS associated with the postpump syndrome. , $^{43,28}$ Picone et $\mathrm{al}^{23}$ demonstrated that the severe lung injury typical of postpump syndrome could be caused by multiple, sequential insults. Their data established that CPB causes pulmonary neutrophil sequestration without lung injury. In the most studied model of sequential insult ARDS, gut ischemia serves as the initial insult and causes pulmonary neutrophil sequestration without lung injury. ${ }^{36-38}$ A subsequent exposure to an otherwise benign insult (LPS $1 \mu \mathrm{g} / \mathrm{kg}$ for $50 \mathrm{~min}$ in this case) activates primed and sequestered neutrophils to cause vascular injury, which leads to postpump syndrome. ${ }^{23,28}$ From our results on cardiorespiratory function, ${ }^{28}$ we suggest that exposure to a benign event (this low dose of LPS is used to reproduce a low level of exposure to infectious agents) aggravates the lung injury initially primed by the CPB procedure. This was corroborated by the greater release of MMPs in BAL and blood, as well as the increase in leukocyte levels in blood in the CPB + LPS group.

Our results may be explained by the different expression patterns of MMP-2 and -9 . Whereas MMP-2 is constitutively 
expressed in various cell types, MMP-9 is strongly induced in epithelial cells by inflammatory cytokines, particularly tumor necrosis factor- $\alpha .{ }^{6,39}$ Inflammatory conditions are also associated with increased activation of oxidant-producing enzymes and NO, which could directly affect MMP expression or activation. ${ }^{19-22}$ The release of MMP-2 from intracellular reserve in plasma and BAL cells may explain the precocious rise of MMP-2 activity in plasma and BAL. The same mechanism could explain the rapid rise of MMP-9 activity in plasma. As a matter of fact, infusion of LPS in healthy volunteers resulted in a rapid increase of plasma MMP-9 activity, likely resulting from liberation of this gelatinase from leukocytes, endothelium, and vascular cells ${ }^{40}$ However, the rise of MMP-9 activity in BAL appeared only after $24 \mathrm{~h}$ in our experiment. This time allows for the induction of expression of MMP-9. ${ }^{39}$ This could also reflect the role of the lung as end organ to present the induced release of MMP-9 in plasma with a lag time in BAL.

Inhaled NO abolished significantly the rise in MMP-2 activity in plasma after 4 and $24 \mathrm{~h}$ and in BAL after $24 \mathrm{~h}$ in the CPB group without effect in sham operation, whereas neither CPB alone or CPB + inhaled NO affected MMP-9. The effect of inhaled NO was marked in the CPB + LPS group, where the rise in gelatinase activity was the highest. In this group, inhaled NO abolished significantly the rise in MMP-2 in both plasma and BAL after 4 and $24 \mathrm{~h}$. The effect of inhaled NO on MMP-9 was not significant in plasma (whereas MMP-9 increased in plasma at T4 for CPB + LPS), and this effect was present in BAL after $24 \mathrm{~h}$. Because inhaled NO did not (or did minimally) influence cell count, cell composition in BAL, and leukocyte and platelet count in blood, these results may be explained by the inhibition of the release from intracellular stores or by a decreased induction of MMPs synthesis by cells. Inhaled NO acted as an anti-inflammatory agent by decreasing neutrophil numbers in BAL and its major chemoattractant, interleukin- $8,{ }^{30}$ as well as MMPs. Inhaled NO also increased cell apoptosis in the lungs during inflammatory conditions, which may explain in part how it resolves pulmonary inflammation. . $9,30^{2}$

Okamoto et $\mathrm{al}^{41}$ demonstrated that the inhibitory effects of $S$-nitrosothiols on MMP-9 expression were associated with diminished nuclear translocation and activation of NF- $\mathrm{kB}$. $\mathrm{NF}-\kappa \mathrm{B}$ activation is involved in the induction of various proinflammatory genes and is critical in inflammatory-immune processes in the lung. Various studies have indicated that NO (or $S$-nitrosothiols) may regulate inflammatory processes by suppressing NF- $\mathrm{\kappa B}$ activation (e.g., in neutrophils or alveolar macrophages). ${ }^{41,42}$ Consequently, the inhibitory effects of $S$ nitrosothiols on MMP-9 expression may be caused by the inhibition of NF- $\mathrm{KB}$ activation, and the effects on MMP-9 are most likely not specific but may be common to other NF-kBregulated genes. ${ }^{41}$

Neither Okamoto et $\mathrm{al}^{41}$ or the present authors have measured the TIMP-1 level in plasma and BAL. To have a complete idea of the regulation of MMP activity, it would have been indicated to do so (but the technology was not accessible at the time of the study), particularly with the recent demonstration that exogenous NO has no modulatory effect on the extracellular TIMP-1 content but strongly amplifies the early increase in cytokine-induced TIMP-1 mRNA and protein levels. ${ }^{42} \mathrm{~A}$ complete measurement of concentrations in MMP2 and MMP-9 as well as TIMP-1 and TIMP-2 associated with a genomic evaluation of the MMP-TIMP pathway could give us a precise idea of the potential modulation of inflammation and subsequent lung injury by inhaled NO during postpump syndrome.

We conclude that MMP-2 and -9 may play a role in the pathogenesis of inflammatory lesions encountered during the use of the CPB procedure, particularly when associated with a postpump syndrome. Furthermore, the release of MMPs appears to be related to the severity of inflammatory stimuli, at least in our model mimicking the clinical entity of postpump syndromes related to multiple, sequential insults. The preemptive, continuous administration of inhaled NO attenuates the rise of MMP-2 and -9 activity related to the inflammatory reaction. These results, in association with the beneficial effects of inhaled NO on oxygenation and pulmonary hemodynamics in the same pig CPB model, ${ }^{28}$ and the reduced postoperative bleeding observed with the combined treatment of inhaled $\mathrm{NO}$ and iloprost ${ }^{34}$ suggest that the preemptive and continuous administration of inhaled NO must be considered as a possible new therapeutic option for patients subjected to CPB. Before that, the exact mechanisms of action must be demonstrated, as well as the clinical use including selection of patients, starting and weaning conditions of $\mathrm{NO}$ inhalation, possible toxicity, and side effects.

\section{ACKNOWLEDGMENTS}

The authors thank G. Deby for her critical review of the manuscript, Ovid Da Silva for editing the manuscript, and Serge Séguin for technical assistance.

\section{REFERENCES}

1. Parks WC, Mecham RP. Matrix Metalloproteinases. San Diego: Academic Press; 1998.

2. Lagente V, Manoury B, Nénan S, et al. Role of matrix metalloproteinases in the development of airway inflammation and remodeling. Braz J Med Biol Res. 2005;38:1521-1530.

3. Shah PK. Inflammation, metalloproteinases, and increased proteolysis: an emerging pathophysiological paradigm in aortic aneurysm. Circulation. 1997;96:2115-2117.

4. Carney DE, Lutz CJ, Picone AL, et al. Matrix metalloproteinase inhibitor prevents acute lung injury after cardiopulmonary bypass. Circulation. 1999;100:400-406.

5. Benjamin IJ. Matrix metalloproteinases: from biology to therapeutic strategies in cardiovascular disease. J Invest Med. 2001;49:381-397.

6. Yao PM, Maitre B, Delacourt C, et al. Divergent regulation of 92-kDa gelatinase and TIMP-1 by HBECs in response to IL-1 $\beta$ and TNF- $\alpha$. Am J Physiol. 1997;273:L866-L874.

7. Asimakopoulos G, Smith PLC, Ratnatunga CP, et al. Lung injury and acute respiratory distress syndrome after cardiopulmonary bypass. Ann Thorac Surg. 1999;68:1107-1115.

8. Taggart DP, el Fiky M, Carter R, et al. Respiratory dysfunction after uncomplicated cardiopulmonary bypass. Ann Thorac Surg. 1993;56: 1123-1128.

9. Gott JP, Cooper WA, Schmidt FE Jr, et al. Modifying risk for extracorporeal circulation: trial of four anti-inflammatory strategies. Ann Thorac Surg. 1998;66:747-754.

10. Rady MY, Ryan T, Starr NJ. Early onset of acute pulmonary dysfunction after cardiovascular surgery: risk factors and clinical outcome. Crit Care Med. 1997;25:1931-1839. 
11. Joffs C, Gunasinghe HR, Multani MM, et al. Cardiopulmonary bypass induces the synthesis and release of matrix metalloproteinases. Ann Thorac Surg. 2001;71:1518-1523.

12. Steinberg J, Fink G, Picone A, et al. Evidence of increased matrix metalloproteinase- 9 concentration in patients following cardiopulmonary bypass. J Extrac orpor Technol. 2001;33:218-222.

13. Mayers I, T Hurst T, Puttagunta L, et al. Cardiac surgery increases the activity of matrix metalloproteinases and nitric oxide synthase in human hearts. J Thorac Cardiovasc Surg. 2001;122:746-752.

14. Torii K, Iida K, Miyazaki Y, et al. Higher concentrations of matrix metalloproteinases in bronchoalveolar lavage fluid of patients with adult respiratory distress syndrome. Am J Respir Crit Care Med. 1997;155:43-46.

15. Cheung PY, Sawicki G, Wosniak M, et al. Matrix metalloproteinase-2 contributes to ischemia-reperfusion injury in the heart. Circulation. 2001; 101:1833-1839.

16. Rossaint R, Gerlach H, Schmidt-Ruhnke H, et al. Efficacy of inhaled nitric oxide in patients with severe ARDS. Chest. 1995;107:1107-1115.

17. De Caterina R, Libby P, Peng HB, et al. Nitric oxide decreases cytokineinduced endothelial activation Nitric oxide selectively reduces endothelial expression of adhesion molecules and proinflammatory cytokines. J Clin Invest. 1995;96:60-68.

18. Kubes P, Suzuki M, Granger DN. Nitric oxide: an endogenous modulator of leukocyte adhesion. Proc Natl Acad Sci U S A. 1991;88: 4651-4655.

19. Mayers I, Salas E, Hurst T, et al. Increased nitric oxide synthase activity after canine cardiopulmonary bypass is suppressed by s-nitrosoglutathione. J Thorac Cardiovasc Surg. 1999;117:1009-1016.

20. Sawicki GE, Salas E, Murat J, H et al. Release of gelatinase A during platelet activation mediates aggregation. Nature. 1997;386:616-619.

21. Mayers I, Hurst T, Radomski A, et al. Increased matrix metalloproteinase activity after canine cardiopulmonary bypass is suppressed by a nitric oxide scavenger. $J$ Thorac Cardiovasc Surg. 2003;125:661-668.

22. Marcet-Palacios M, Graham K, Cass C, et al. Nitric oxide and cyclic GMP increase the expression of matrix metalloproteinase-9 in vascular smooth muscle. JPET. 2003;307:429-436.

23. Picone A, Lutz C, Finck C, et al. Multiple sequential insults cause postpump syndrome. Ann Thorac Surg. 1999;67:978-985.

24. Nieman G, Searles B, Carney D, et al. Systemic inflammation induced by cardiopulmonary bypass: a review of pathogenesis and treatment. $J$ Extrac orpor Technol. 1999;31:202-210.

25. Hubert M, Salazkin I, Desjardins J, et al. Cardiopulmonary bypass surgery in swine: a research model. J Exp Anim Sci. 2003;43:135-149.

26. Shah N, Nakayama D, Jacob T, et al. Efficacy of inhaled nitric oxide in a porcine model of adult respiratory distress syndrome. Arch Surg. 1994; 129:158-164.

27. Dube L, Francoeur M, Troncy E, et al. Comparison of two administration techniques of inhaled nitric oxide on nitrogen dioxide production. Can J Anaesth. 1995;42:922-927.
28. Troncy E, Hubert B, Pang D, et al. Pre-emptive and continuous inhaled NO counteracts the cardiopulmonary consequences of extracorporeal circulation in a pig model. Nitric Oxide Biol Chem. 2006;14: 261-271.

29. El Kebir D, Taha R, Hubert B, et al. The anti-inflammatory effect of inhaled nitric oxide on pulmonary inflammation in a swine model. Can J Physiol Pharmacol. 2005;83:252-258.

30. El Kebir D, Hubert B, Taha R, et al. Effects of inhaled nitric oxide on inflammation and apoptosis after cardiopulmonary bypass. Chest. 2005; 128:2910-2917.

31. Sawicki G, Sanders E, Salas E, et al. Localization and translocation of MMP-2 during aggregation of human platelets. Thromb Haemost. 1998; $80: 836-839$.

32. Fox-Robichaud A, Payne D, Hasan S, et al. Inhaled NO as a viable antiadhesive therapy for ischemia-reperfusion injury of distal microvascular beds. J Clin Invest. 1995;96:60-68.

33. Samama C, Diaby M, Fellahi J, et al. Inhibition of platelet aggregation by inhaled nitric oxide in patient with acute respiratory distress syndrome. Anesthesiology. 1995;83:56-65.

34. Chung A, Wildhirt S, Wang S, et al. Combined administration of nitric oxide gas and iloprost during cardiopulmonary bypass reduces platelet dysfunction: a pilot clinical study. J Thorac Cardiovasc Surg. 2005;129: 782-790.

35. Gianetti J, Del Sarto P, Bevilacqua S, et al. Supplemental nitric oxide and its effect on myocardial injury and function in patients undergoing cardiac surgery with extracorporeal circulation. J Thorac Cardiovasc Surg. 2004; 127:44-50.

36. Anderson B, Moore E, Banerjee A. Phospholipase A2 regulates critical inflammatory mediators of multiple organ failure. J Surg Res. 1994;56: 199-205.

37. Kim F, Moore E, Moore F, et al. Reperfused gut elaborates PAF that chemoattracts and primes neutrophils. J Surg Res. 1996;58:636-640.

38. Waisman D, Brod V, Dickstein R, A et al. Effects of inhaled nitric oxide on lung injury after intestinal ischemia-reperfusion in rats. Shock. 2005;23: $150-155$.

39. Hozumi A, Nishimura Y, Nishiuma T, et al. Induction of MMP-9 in normal human bronchial epithelial cells by TNF-alpha via NF-kappa B-mediated pathway. Am J Physiol Lung Cell Mol Physiol. 2001;281: L1444-L1452.

40. Albert J, Radomski A, Soop A, et al. Differential release of matrix metalloproteinase-9 and nitric oxide following infusion of endotoxin to human volunteers. Acta Anaesthesiol Scand. 2003;47:407-410.

41. Okamoto T, Valacchi G, Gohil K, et al. $S$-nitrosothiols inhibit cytokinemediated induction of matrix metalloproteinase- 9 in airway epithelial cells. Am J Respir Cell Mol Biol. 2002;27:463-473.

42. Akool E, Doller A, Müller R, et al. Nitric oxide induces TIMP-1 expression by activating the transforming growth factor $\beta$-Smad signaling pathway. J Biol Chem. 2005;280:39403-39416. 